\title{
Processing of new arguments at clause boundaries
}

\author{
KARL HABERLANDT \\ Trinity College, Hartford, Connecticut \\ and \\ ARTHUR C. GRAESSER \\ Memphis State University, Memphis, Tennessee
}

\begin{abstract}
In a subject-paced reading-time study, we examined the processing of new arguments at clause boundaries. Word reading times increased with the cumulative number of new-argument nouns at clause boundaries (as well as at sentence boundaries). New-argument nouns had a greater impact at clause boundaries than at nonboundary locations. In accordance with a bufferintegrate-purge model of reading (see Jarvella, 1979), the increase of reading times at boundaries was attributed to the integration of new information from the current sentence with prior information in the text representation. The increase at nonboundary locations was attributed to the growing load of buffering the new information. Reading times at clause boundaries were influenced to a greater extent by text-level integration than by such sentence-level processes as organization of words into clauses and linking of clauses within a sentence. The new contribution of this study was that it showed that clause boundaries provide an opportunity not only for sentence-level processes, but also for a text-level process, namely, the integration of text-new information with the growing text representation.
\end{abstract}

In this study, we examined text-level processes at clause boundaries. In the past, the clause boundary has been identified by psycholinguists as the location for a variety of parsing and interpretation processes at the sentence level. To some extent, these processes result from the constraints of the human information-processing system, specifically of working memory. The relatively small capacity of working memory imposes a limit on the number of individual words a reader can keep in mind at any one time. As a consequence, words are remembered verbatim only until they can be chunked into a clause. At clausal boundaries important semantic information is retained, whereas individual words and surface structure details are less likely to be remembered (e.g., Aaronson \& Scarborough, 1976, 1977; Clark \& Clark, 1977; Fodor, Bever, \& Garrett, 1974; Glanzer, Fischer, \& Dorfman, 1984; Jarvella, 1979; Johnson \& Mandler, 1980; Just \& Carpenter, 1987; Miller \& Kintsch, 1980; Perfetti \& Goldman, 1976; van Dijk \& Kintsch, 1983).

The role of clausal structure in sentence comprehension has been illustrated by at least three lines of research: recall studies (Jarvella, 1979), click studies (Green, 1977), and reading-time studies (Aaronson \& Scarborough,

\footnotetext{
This research was supported in part by NSF Grant BNS-8410037 and by NICHD Grant 1 R15 HD23121-01. We thank Peter Bradley, Judith Kiely, and Kenneth Lowry for their assistance with this research. We also thank Alice Healy, Ernst Z. Rothkopf, Noel Sharkey, and an anonymous reviewer for their comments on an earlier version of this paper. Requests for reprints should be sent to Karl Haberlandt, Department of Psychology, Trinity College, Hartford, CT 06106.
}

1976). Jarvella (e.g., 1979) showed that recall of verbatim information is nearly perfect for the most recent clause, supporting the claim of a verbatim trace in working memory. Chang (1980) observed a similar result in a recognition study. This result is precisely what one would expect from the clausal recoding theory (see Aaronson \& Scarborough, 1977; Fodor et al., 1974; van Dijk \& Kintsch, 1983). The load of interpreting, recoding, and chunking leads to an increase in word reading times at the final word of the clause (Aaronson \& Scarborough, 1976), and it reduces the processing capacity for such secondary tasks as monitoring acoustic clicks (Green, 1977).

Two types of clausal comprehension processes have been distinguished: intraclause organization and interclause linking (Aaronson \& Scarborough, 1976). Intraclause organization consists of interrelating the words within a clause and recoding the words into semantic clausal chunks. Interclause linking connects clauses from a sentence via argument overlap and via bridging inferences, resulting in a representation of the entire sentence. Other theorists also acknowledge these organization and linking processes within sentences, although their terminologies may differ. For example, van Dijk and Kintsch (1983) subsumed processes akin to intracluase organization and interclause linking under the term propositional strategies. These are semantic processes that relate the predicate of an atomic proposition with the corresponding arguments, and form complex propositions from atomic propositions (see also Clark \& Clark, 1977; Just \& Carpenter, 1987; Perfetti, 1985). 
It is widely recognized that reading comprehension is based on a number of component processes, including sentence-level and text-level operations. Among the sentence-level processes, the organization and linking processes discussed above have been documented for sentences presented in isolation without context. These processes have been shown to be concentrated at clausefinal words and to produce a cognitive load beyond that required for processing at the word level, such as lexical access (Aaronson \& Scarborough, 1977).

Text-level processes produce a text representation that contains the semantic content of the text along with bridging inferences for establishing text coherence. This representation is constructed in an incremental manner, yielding a larger structure with additional sentences (Collins, Brown, \& Larkin, 1980; Foss, 1982; Gernsbacher, 1985; Olson, Mack, \& Duffy, 1981; van Dijk \& Kintsch, 1983). The text-level processes include operations that relate the incoming sentence to the prior passage context. Text-level processing assumes, of course, that readers distinguish between text-new and old (or "given") information (Clark \& Clark, 1977; Haviland \& Clark, 1974; Kieras, 1984).

A buffer-integrate-purge model (Jarvella, 1979) specifies how text comprehension can proceed in an incremental and cumulative manner, and yet fall within the constraints of a working memory with limited capacity. During the buffering phase of the cycle, the words within a clause accumulate in a temporary buffer until the end of a clause or sentence is reached. During the integration phase at the end of a clause or a sentence, the new information in the clause is linked with the previous passage context and thus is passed to the text representation. Integration processes typically involve such operations as a search for antecedents and/or inferences. They are relatively resource-intensive and time-consuming, at least compared with buffering operations (Sharkey \& Sharkey, 1987). At the conclusion of a cycle during the purge phase, the buffer is cleared of the content in the current clause.

The present study examined the relation between textnew information and clausal processing with respect to the buffer-integrate-purge model. The amount of new information is scaled by computing the number of newargument nouns that accumulate prior to a clause boundary. A new-argument noun is a noun that occurs for the first time in a text. We have selected new-argument nouns as a measure of new information because it is easier and more reliable to scale the given-new status of nouns than of such predicates as verbs, adjectives, and connectives. In addition, new-argument nouns have been a robust predictor of processing time. For example, they take longer to process than repeated nouns (Kintsch, Kozminsky, Streby, McKoon, \& Keenan, 1975), and reading times increase with the cumulative number of new arguments both prior to the sentence boundaries and at the boundaries themselves (Haberlandt \& Graesser, 1985; Haberlandt, Graesser, Schneider, \& Kiely, 1986). The increase was steeper at the boundary words than at the nonboundary words. The increase at the boundary was attributed to integration processes, whereas the smaller increase at nonboundary words was attributed to buffering processes.

The increase of reading times of boundary words with the cumulative number of arguments would be predicted by most theories of comprehension. Clearly, the timeconsuming processes of integrating ideas within a clause and between clauses should be further increased when new referents must be constructed in the text representation. The increase in reading times for nonboundary words can be explained in at least two ways. According to the first account, the reader does not always wait for the end of clauses to invoke integration; integration may occasionally be invoked from within a clause. According to the second explanation, reading slows down as a function of the cognitive load in the buffer, and that load increases directly with the cumulative number of new-argument nouns held in the buffer. The second explanation would presuppose a smart or sophisticated buffer (Sharkey \& Sharkey, 1987) that takes account of the prior context of any noun and thus distinguishes between the new or old status of a noun.

The goal of this research was to evaluate predictions of the buffer-integrate-purge model for clausal boundaries, as well as for sentence boundaries. Based on our previous research, we predicted an interaction between the cumulative number of new arguments per clause and word position (Haberlandt \& Graesser, 1985; Haberlandt et al., 1986). Two word positions were distinguished, nonboundary and boundary positions. Word reading times were expected to increase with the cumulative number of new arguments at both nonboundary and boundary locations. The increase should be steeper at clause boundaries because integration was assumed to require more cognitive resources per new argument than buffering would demand. The load at the clause boundaries should be a result of integrating the new arguments with prior information in the text representation. In contrast, the increase in load at nonboundary words should reflect the memory load added by each new-argument noun.

The moderate length of sentences in our previous research ( $M=13.12$ words) may have induced in our readers a strategy of focusing on the sentence boundaries. The present research used relatively long passages and sentences. In addition to enabling us to examine intersentence integration at clause boundaries, the use of long sentences enabled us to examine whether readers are able to postpone intersentence integration to the end of the sentence.

We used natural passages, one expository and one narrative. The passages were sufficiently long to provide an observation base of over 200 clauses embedded in a natural text. We selected realistic passages because we wanted to be able to draw conclusions about comprehension as it occurs in instructional reading situations (e.g., Rothkopf \& Billington, 1983). The two passage types 
were selected simply to evaluate the generality of the predictions. Similarly, for purposes of generality, we used two task conditions: question-answer and summarization. Although text type and task were manipulated, we were not directly interested in such comparisons. Instead, the focus of the present research was on intersentence integration at clause and sentence boundaries, as well as at nonboundary locations.

\section{METHOD}

\section{Subjects}

The subjects were 96 undergraduates at Trinity College who participated in the experiment in fulfillment of a course requirement.

\section{Materials}

We used one short practice passage and two experimental passages, an exposition and a narrative. The expository passage was a shortened version of a Scientific American article about the surface of Mars (Arvidson, Binder, \& Jones, 1978). The narrative was a Reader's Digest article about the adventures of John Colter in the Wild West (Blevins, 1976; see also Just \& Carpenter, 1987; Just, Carpenter, \& Masson, 1982). The Colter passage consisted of 121 sentences averaging $16.5(S D=10.40)$ words. The mean number of new arguments per sentence was $2.27(S D=2.65)$, where a new-argument noun was a noun that appeared for the first time in a text. The Mars passage had 112 sentences, averaging 23 (SD = $8.58)$ words and $1.95(S D=1.98)$ new arguments per sentence. The Colter passage contained 2,014 words and the Mars passage 2,605 words. In the Mars passage, the length of words was greater and their occurrence frequency was less than in the Colter passage. In the Mars passage, the mean word length was 5.06 letters ( $S D$ $=2.69$ ), and the mean log occurrence frequency was 2.66 ( $S D=$ 1.50 ), according to the count by Kucera and Francis (1967). The corresponding values for the Mars passage were $4.46(S D=2.29)$ and $2.79(S D=1.40)$.

For each word position, the cumulative number of new-argument nouns per sentence and per clause was determined. The cumulative new-argument variable was coded separately for clauses and for sentences. For example, for clauses, all words preceding the occurrence of the first new-argument noun within the clause received a code of 0 ; following the first new-argument noun, all words up to the occurrence of the second new noun were given a 1 ; and so forth. At the beginning of a new clause, the cumulative count was reset to 0 . Thus each word in the clause was categorized in terms of the number of new arguments up to that point. A similar coding of new-argument nouns was performed in terms of sentence boundaries.

We distinguished two types of clauses: main clauses and modifying clauses. Every sentence has one or more main clauses, and zero or more modifying clauses. A modifying clause embellishes a word or phrase in another clause, often before the other clause is completed. The modifying clause cannot stand alone, being dependent on another clause. In contrast, a main clause stands alone in the sense that the expression can apparently be interpreted when the end of the main clause is reached. The present study coded final words of main clauses, but not final words of modifying clauses.

A main clause has a subject and a predicate. A subject is a noun phrase; a predicate is a verb expression and often contains some additional noun phrases. Sentence 1 is a sample sentence from our texts with a single main clause: (1) "Mariner 9 photographed practically the entire surface of Mars at a resolution of a few kilometers." Sentences may contain two or more main clauses that are linked by connectives (e.g., and, then, because, etc.). In most of the present sentences containing multiple main clauses, the subject and predicate of the first clause were completed before the second clause began, as illustrated in Sentence 2: (2) "All this way he must have been meeting bands of Crows / for he was ambling through some of their favorite country." Crows marks the end of the first main clause in Sentence 2. On the other hand, words concluding clauses that were not ready to be interpreted were not included in the set of clause-final words. For example, Sentence 3 illustrates the case of a subordinate clause not ready to be fully interpreted when the end-of-clause word is reached: (3) "Because he was ambling through some of their favorite country, all this way he must have been meeting bands of Crows."

Excluding the sentence boundaries, there were 130 main-clause boundaries in the Colter passage, and 89 in the Mars passage. Of the total of 233 sentences, 87 sentences contained no additional main clause. There were 146 sentences with at least one additional main clause. Of these, 53 sentences contained two or more additional main clauses.

\section{Apparatus}

Stimulus presentation and reading-time measurements were controlled by an IBM PC computer. Words were presented on the screen according to the moving-window method (e.g., Just, Carpenter, \& Woolley, 1982). Words were presented one at a time on a video terminal. When text presentation began, the screen was filled with strings of dashes, corresponding to words, and spaces in the normal layout of the text. A reader proceeded through the text by pressing a key. With each keypress, a word was presented on the screen, replacing the dashes and removing the previous word. Thus, only one word was visible at any given time. The reading time of a word was defined as the interval between successive keypresses. This method produces longer reading times than the eye fixation method and does not allow regressions by the reader to previous text. Nevertheless, the reading-time patterns from both methods are similar (see Just, Carpenter, \& Woolley, 1982, p. 231). Also, as in natural reading, the eye moves from left to right and makes return sweeps when advancing to the next line.

\section{Procedure}

In both the comprehension and summarization conditions, each subject first read the practice passage. Forty-eight of the subjects then read the Colter passage, followed by the Mars passage. The other 48 subjects received the experimental passages in the opposite order. The subjects were instructed to read each passage carefully. Each passage was presented in five sections of $250-700$ words, followed by a pause of $15 \mathrm{sec}$. Sections started with headings of 2-7 words, which were not analyzed. After reading a passage, subjects in the comprehension condition answered 32 true-false questions presented successively on the screen. In contrast, the summarization subjects were instructed to provide a brief summary of the passage just read by speaking into a cassette recorder. There were 48 students in each of the two conditions. An experimental session lasted approximately $50 \mathrm{~min}$ in the comprehension condition and $55 \mathrm{~min}$ in the summarization condition.

\section{RESULTS}

The primary goal of this research was to investigate textlevel integration and buffering relative to clause boundaries. We documented such integration by new-argument effects at clausal boundaries and at nonboundary words. Because both sentence-level and text-level processes occur at clause boundaries, we also examined which of these processes exerts a greater influence on the reading times of clause-final words. Specifically, in one analysis we compared intraclause organization and text-level integration. In a separate analysis we compared interclause link- 
ing and integration. Finally, we examined integration processes at the sentence boundary and whether or not they were influenced by the presence of additional clause boundaries in the sentence.

\section{Intersentence Integration at Clause Boundaries}

In a preliminary regression analysis, we found that reading times at clause boundaries were $27 \mathrm{msec}$ longer than at nonboundary positions $(t=7.94)$. The dependent variable was the set of 4,572 word reading times averaged across 48 readers from both conditions. The stimulus factors partialed out in this analysis included word-level, sentence-level, text-level, and layout factors. The wordlevel variables included word length and the logarithm of the word occurrence frequency. The sentence-level variables included the beginning of sentences, the end of sentences, and clausal boundaries. The text-level variables included passage type, cumulative number of newargument nouns, and sentence-serial position within the passage. There were also the following four layout variables: beginning of line, end of line, beginning of screen, and end of screen. ${ }^{1}$

This clausal effect replicates similar results observed by Aaronson and Scarborough (1977) and by Haberlandt and Graesser (1985). However, by itself it does not constitute support for buffering and integration processes. If the buffer-integrate-purge model were valid for clausal boundaries, we would expect a position $X$ new argument interaction relative to the clause boundary. Any increase in reading times with the cumulative number of newargument nouns should be steeper for clause-final words than for nonboundary words.

We examined clausal processing as a function of new arguments in a three-way analysis of variance (ANOVA) on the reading times of content words at clausal boundaries (excluding the sentence boundary) and at nonboundary locations. The three within-subjects factors were passage type (Colter vs. Mars), word position (boundary words vs. nonboundary words), and cumulative number of new arguments per clause. There were three levels of the new-argument variable: zero, one, and two or more new arguments per clause. This analysis yielded the predicted interaction between position and cumulative new arguments $\left[F(2,190)=10.17, M S_{\mathrm{e}}=4,114\right]$. In addition, there were significant main effects for position $\left[F(1,95)=38.82, M S_{\mathrm{e}}=21,924\right]$ and cumulative number of new arguments $\left[F(2,190)=116.19, M S_{\mathrm{e}}=8,343\right]$. In a simple-effects analysis, we found that the newargument effect was significant for nonboundary locations $\left[F(2,190)=103.60, M S_{\mathrm{e}}=2,990\right]$, as well as for boundary locations $\left[F(2,190)=74.10, M S_{\mathrm{e}}=9,468\right]$. There was also a significant main effect for passage type $[F(1,95)$ $\left.=5.99, M S_{\mathrm{e}}=41,045\right]$, which resulted from longer reading times for the expository Mars passage (see also Graesser, Hoffman, \& Clark, 1980).

The position $\times$ new argument interaction, together with significant simple effects for new arguments at each position, validates the distinction between buffering and intersentence integration relative to clause boundaries. We had previously demonstrated these processes at the sentence boundary; now we have evidence for a similar scenario at the clause boundary (see Figure 1).

The cumulative new-argument effects described thus far show that the clausal boundary provides an occasion for text-level processes. Of course, processing at clause boundaries is also influenced by intrasentence processing, as noted in the introduction.

\section{Text-level Processing versus Sentence-level Processing at Clause Boundaries}

Our next set of analyses compared sentence-level processing with text-level processing at clause boundaries. In these analyses, intersentence integration was measured by the number of new arguments in specified clauses. Text-level processing was compared separately with each of the two sentence-level processes introduced above: intraclause organization and interclause linking. Intraclause organization chunks the words of the current constituent into a unified clause. Interclause linking refers to the connecting of information from the current clause with information from prior clauses of the same sentence. Aaronson and Scarborough (1977) used the number of words in specific clauses as an index for the two processes. New arguments were not used in that study because they are a text-level measure rather than a sentence-level measure.

We evaluated the intraclause organization load as follows. We selected the 146 sentences with two or more clauses and analyzed the reading times of the final words of first clauses only. The reason for using only first clauses was that, in addition to intraclause organization, there is some clausal linking at the boundaries of subsequent clauses. Therefore, it would have been difficult to estimate intraclause organization per se. Thus, this analysis examined which of the following was a better predictor of the reading times of final words of first clauses:

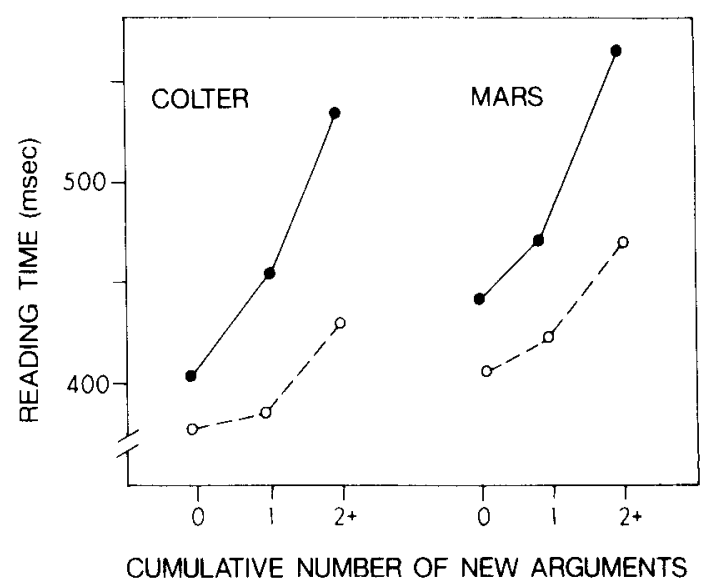

Figure 1. Reading times of clause-final words (solid lines) and nonboundary words as a function of passage and the number of new arguments per clause. 
(1) intraclause organization, as indexed by the number of words, or (2) intersentence integration, as indexed by the number of new argument nouns.

For each of the 96 subjects, two sets of regression analyses were performed on the reading times of the $146 \mathrm{fi}_{1}$ nal words of first clauses, with the number of words or the number of new-argument nouns as the respective predictor variable of interest. In both analyses, the remaining variables were sentence-serial position, word length, and word occurrence frequency. Data from individuals were used to meet the criticism that computing regressions on average reading times inflates experimental effects (Kliegl, Olson, \& Davidson, 1982). The standardized regression (beta) coefficients ${ }^{2}$ derived from each individual regression were analyzed as one would analyze any dependent variable in an ANOVA (see Haberlandt, Berian, \& Sandson, 1980; for an alternative approach see Pedhazur, 1977). We submitted the beta coefficients for the number of words versus the new-argument noun variable to an ANOVA, with passage type as an additional within-subjects factor. Text-level integration was more prominent than sentence-level organization at the clause boundary. This was supported by greater beta values for the number of new-argument nouns per clause $(M=0.117)$ than for the number of words per clause $(M=0.029)$ $\left[F(1,95)=64.86, M S_{\mathrm{e}}=0.01\right]$.

The comparison of text-level integration with interclause linking within a sentence was based on 53 sentences that contained three or more main clauses. This analysis focused on boundaries of second clauses. The specific question was whether more processing was devoted to linking the first clause with the second clause, or to integrating the new arguments of the second clause with prior sentences. The index for any linking occurring at the second clause was the number of words in the first clause (e.g., Aaronson \& Scarborough, 1977); text-level integration was measured by the number of new arguments in the second clause. The number of arguments in the second (current) clause was chosen because the buffer-integratepurge model predicts that integration occurs as soon as the current clause is completed. For each of the 96 subjects, we performed two sets of regression analyses on the reading times of 53 final words of second clauses with the number of words or number of new-argument nouns as the critical predictor variable. The remaining variables were sentence-serial position, word length, and word $\alpha c$ currence frequency. The beta coefficients for the number of words versus the new-argument noun variable were submitted to an ANOVA, with passage type as an additional within-subjects factor. As in the previous ANOVA, the beta value for number of new argument nouns per clause $(M=0.109)$ was significantly greater than for the number of words per clause $(M=0.04)[F(1,95)=$ $\left.12.70, M S_{\mathrm{e}}=0.04\right]$.

These comparisons showed that text-level processes were a better predictor of clausal reading times than such sentence-level processes as the organization of words into clausal chunks and the linking of clauses within sentences.
There were no interactions with passage in either comparison, which indicates that intersentence integration was stronger than either intrasentence process independent of passage type. This interpretation assumes that the estimates of intrasentence processing we used are applicable to our experimental situation, although there were differences between our experiment and Aaronson and Scarborough's (1977) study. In sum, our results demonstrate text-level integration at clause boundaries and buffering of new information prior to the clause boundaries.

\section{End-of-Sentence Processing}

The sentences in the Mars and Colter passages contained an average of 19.62 words, compared with 13.12 words in our previous research (Haberlandt et al., 1986). It is by no means certain that readers would be capable of postponing integration processes until the sentence boundary with sentences $50 \%$ longer than we had used previously. The results show, however, that readers did perform some integration processing at the sentence boundaries even with the longer sentences used here.

Figure 2 shows the mean reading times for sentencefinal words and nonboundary words, which were words located at neither sentence nor clause boundaries. The reading times are plotted as a function of the cumulative number of new arguments in the two passages. As in our previous research (Haberlandt et al., 1986), reading times increased for sentence-boundary words and nonboundary words with an increasing amount of new information in the sentence. The increase was steeper at the sentence boundary than at nonboundary locations, as predicted by the buffer-integrate-purge model. This was confirmed in a $2 \times 2 \times 4$ ANOVA on the data shown in Figure 2 . The factors were passage type (Colter vs. Mars), word position (boundary vs. nonboundary), and the number of new arguments per sentence $(0,1,2$, and $3+)$. The interaction involving position and new arguments was significant $\left[F(3,93)=6.53, M S_{\mathrm{e}}=50,303\right]$

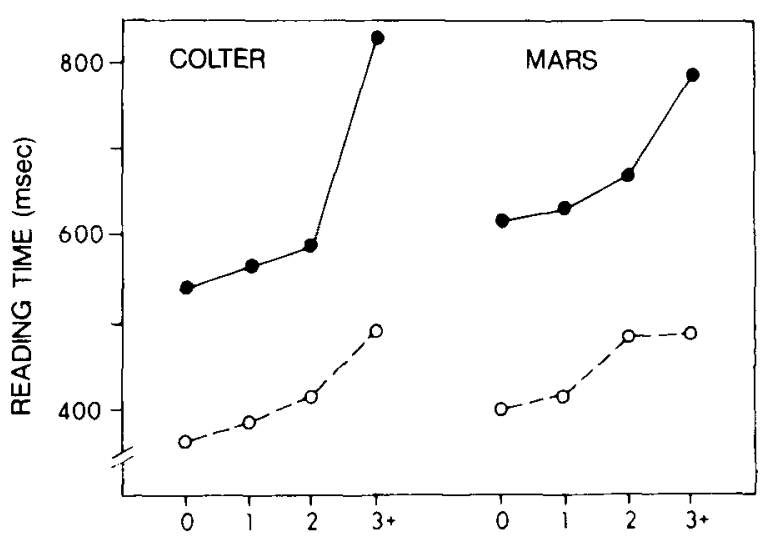

CUMULATIVE NUMBER OF NEW ARGUMENTS

Figure 2. Reading times of sentence-final words (solid lines) and nonboundary words as a function of passage and the number of new arguments per sentence. 
The ANOVA also yielded a significant main effect for position $\left[F(1,95)=40.62, M S_{\mathrm{c}}=439,690\right]$ and a significant new-argument effect $\left[F(3,285)=33.20, M S_{\mathrm{e}}=\right.$ $62,284]$. Passage type and the number of arguments interacted significantly $\left[F(3,285)=5.69, M S_{\mathrm{e}}=30,823\right]$, indicating a different reading-time profile for the two passages. Subsidiary analyses revealed that this difference was attributable to lexical factors. ${ }^{3}$

\section{End-of-Sentence Processing Considered in Light of Clausal Processing.}

Each sentence contained at least one main clause and thus provided at least one opportunity for text-level integration. Our next analysis concerned the effect of additional clauses in a sentence on intersentence integration at the sentence boundary. Because intersentence integration occurred both at clause boundaries and at sentence boundaries, we wondered whether there would be reduced end-of-sentence processing in those 146 sentences that contained additional main clauses. We would expect this reduction from our previous result, which was based on relatively short passages (Haberlandt \& Graesser, 1985 , Figure 1). Thus, we compared the amount of end-ofsentence processing in the 146 sentences with at least one additional main clause with that in the 87 sentences with no additional main clauses. We used the beta coefficient associated with sentence-final words as an index for integration load at the sentence boundary. Partialling out other factors, we found, as predicted, reduced beta coefficients for end-of-sentence processing in sentences that contained additional main clauses $(M=0.065)$ as opposed to sentences without additional clauses $(M=0.108)$. This difference was significant in an ANOVA with passage type as an additional within-subjects factor $[F(1,95)=$ 39.36, $\left.M S_{\mathrm{e}}=0.004\right]$.

\section{DISCUSSION}

We found evidence of intersentence integration at clause and sentence boundaries. At both boundary types, reading times increased monotonically with the cumulative number of new arguments. At nonboundary locations, reading times also increased with the cumulative number of new arguments, but to a lesser degree. These results illuminate three important issues: the new-argument effect, the nature of clausal processing, and the relation between clause boundaries and sentence boundaries.

\section{New-Argument Effects}

Haviland and Clark (1974) reported that the reader devotes more cognitive resources to new information in a text than to old information. This was reflected in longer reading times for sentences containing new information. Kintsch et al. (1975) refined Haviland and Clark's results by demonstrating a linear relationship between text reading time and the number of new arguments in the text (see also Graesser et al., 1980; Haberlandt et al., 1980). Although these aggregate results offered important in- sights on integration processes, they did not reveal the location(s) of integration processing within the sentence.

On the other hand, the pattern of word reading times reported here and in our previous research (Haberlandt \& Graesser, 1985) shows three different components of the new-argument effect and their locations. First, there is an absolute increase in the reading times of newargument nouns resulting from lexical processing and from foregrounding of the new argument. ${ }^{4}$ Second, there is an additional increase in reading times with each added new argument prior to clause boundaries. This confirms the sophisticated buffer hypothesis (Sharkey \& Sharkey, 1987), which assumes that new arguments can be distinguished from old arguments in a memory buffer. It is the added load of maintaining the new information that produces the cumulative increase in the reading time. Third, at the clause boundary, reading times increase with the number of new arguments in the current text segment. The new-argument effect at the boundary reflects those processes that result in the attachment of the new information to antecedent information in the text representation. Such attachment occurs either through reinstatement of the prior information or through bridging inferences. The new-argument effects reported here support the buffer-integrate-purge model (Jarvella, 1979), with the reading time increase at nonboundary locations reflecting the buffering load, and the increase at boundary locations reflecting the integration load.

\section{Processing at Clause Boundaries}

The increase of reading times at clause-final words replicates similar results from research using different paradigms and stimulus materials. For example, Aaronson and Scarborough $(1976,1977)$ reported such a clausal effect with individual sentences in a stationary window situation, and Mitchell and Green (1978, Experiment 4) described the clausal effect in a subject-paced reading experiment using a passage of over 1,000 words. Those results were attributed to sentence-level processes, including interpretation, intraclause organization, and interclause linking. Interpretation involves the identification and segmentation of the surface constituents through a variety of strategies and the construction of propositions (e.g., Clark \& Clark, 1977; see also Frazier, 1983; Marcus, 1980). Intraclause organization includes the processes that interrelate words in a clause and form a coherent semantic chunk (Aaronson \& Scarborough, 1977). Interclause linking results in a complex structure that contains the current clause and previous clauses in a sentence. These processes are concentrated at clause boundaries and produce longer processing times and a concomitant decrease in the performance of secondary tasks (Green, 1977). The new-argument effect at clause boundaries shows that, in addition to these three sentencelevel processes, there is also text-level processing at the clause boundaries. Indeed, by definition, a new argument is a text-level construct. The new-argument effect reflects a "continuity" building process that relates current in- 
formation to prior information in a text (e.g., Jarvella, 1979).

\section{What is the Relation Between Clause Boundaries and Sentence Boundaries?}

The presence of sentence-level and text-level processes at both boundaries suggests that the two boundary types are essentially similar. On the other hand, the two boundaries could be viewed as fundamentally different, because the sentence boundary is final and unambiguous and provides the opportunity for special "sentence wrap-up" processes. Our results favor the view that the boundaries are similar, because the new-argument effect was present at both types of boundaries. Indeed, there was a distribution of integration processes across boundaries within a sentence. Sentences with several clauses provided more opportunities for integration, thus resulting in reduced integration processes at the end of the sentence.

In conclusion, the new contribution of this research is to demonstrate that clausal processing time is not merely greater than the processing time at nonboundary locations, but that it is determined by a text-level factor, namely the integration of text-new information with the reader's text representation. Connections between text-new information and prior information occur at many levels in reading. They occur when atomic propositions are formed by relating a predicate to its arguments. Connections are also made in linking atomic propositions to form a complex propositional structure that frequently transcends clausal boundaries. The connecting process studied here involved the integration of new-argument nouns with prior information across sentence boundaries. Whatever the level of the connecting process, it operates under the limitations of working memory, which permits only a few items to be held concurrently. At each level, information is recoded into more abstract information in order to be retained.

\section{REFERENCES}

aAronson, D., \& Scarborough, H. S. (1976). Performance theories for sentence coding: Some quantitative evidence. Journal of Experimental Psychology: Human Perception \& Performance, 2, 56-70.

Aaronson, D., \& SCARBorough, H. S. (1977). Performance theories for sentence coding: Some quantitative models. Journal of Verbal Learning \& Verbal Behavior, 16, 277-304.

Arvidson, R. E., Binder, A. B., \& Jones, K. L. (1978, March). The surface of Mars. Scientific American, pp. 76-77, 80-89.

Blevins, W. (1976, October). John Colter, greatest of the mountain men. Readers' Digest, pp. 81-82, 86-87, 91-92.

CHang, F. R. (1980). Active memory processes in visual sentence comprehension: Clause effects and pronominal reference. Memory \& Cognition, 8, 58-64.

Clark, H. H., \& Clark, E. V. (1977). Psychology and language: An introduction to psycholinguistics. New York: Harcourt Brace Jovanovich.

Collins, A., Brown, J. S., \& Larkin, K. M. (1980). Inference in text understanding. In R. J. Spiro, B. C. Bruce, \& W. F. Brewer (Eds.), Theoretical issues in reading comprehension (pp. 385-407). Hillsdale, NJ: Erlbaum.

Fodor, J. A., Bever, T. G., \& Garrett, M. F. (1974). The psychology of language: An introduction to psycholinguistics and selective grammar. New York: McGraw-Hill.
Foss, D. J. (1982). A discourse on semantic priming. Cognitive Psychology, 14, 590-607.

Frazier, L. (1983). Processing sentence structure. In K. Rayner (Ed.), Eye movements in reading: Perceptual and language processes (pp. 215-236). New York: Academic Press.

GERNSBACHER, M. A. (1985). Surface information loss in comprehension. Cognitive Psychology, 17, 324-363.

Glanzer, M., Fischer, B., \& Dorfman, D. (1984). Short-term storage in reading. Journal of Verbal Learning \& Verbal Behavior, 23, 467-486.

Graesser, A. C., \& Haberlandt, K. F. (1986). Research on component processes in reading: Reply to Danks. Journal of Experimental Psychology: General, 115, 198-200.

Graesser, A. C., Hoffman, N. L., \& Clark, L. F. (1980). Structural components of reading time. Journal of Verbal Learning \& Verbal Behavior, 19, 135-151.

GrEEN, D. W. (1977). The immediate processing of sentences. Quarterly Journal of Experimental Psychology, 29, 1-12.

Haberlandt, K., Berian, C., \& Sandson, J. (1980). The episode schema in story processing. Journal of Verbal Learning \& Verbal Behavior, 19, 635-650.

Haberlandt, K. F., \& Graesser, A. C. (1985). Component processes in text comprehension and some of their interactions. Journal of $E x$ perimental Psychology: General, 114, 357-374.

Haberlandt, K. F., Graesser, A. C., Schneider, N. J., Kiely, J. (1986). Effects of task and new arguments on word reading times. Journal of Memory \& Language, 25, 314-322.

Haviland, S. E., \& Clark, H. H. (1974). What's new? Acquiring new information as a process in comprehension. Journal of Verbal Learning \& Verbal Behavior, 13, 515-521.

JARVELLA, R. J. (1979). Immediate memory and discourse processing. In G. H. Bower (Ed.), The psychology of learning and motivation: Advances in research and theory (Vol. 13, pp. 379-421). New York: Academic Press.

Johnson, N. S., \& MANDler, J. M. (1980). A tale of two structures: Underlying and surface forms in stories. Poetics, 9, 51-86.

Just, M. A., \& CaRPENTER, P. A. (1987). The psychology of reading and language comprehension. Newton, MA: Allyn and Bacon.

Just, M. A., Carpenter, P. A. \& Masson, M. (1982). What eye fixations tell us about speed reading and skimming (Tech. Rep. March 1982). Pittsburgh, PA: Carnegie-Mellon University.

Just, M. A., Carpenter, P. A., \& Woolley, J. D. (1982). Paradigms and processes in reading comprehension. Joumal of Experimental Psychology: General, 3, 228-238.

KIERAS, D. E. (1984). A method for comparing a simulation to reading time data. In D. Kieras \& M. Just (Eds.), New methods in comprehension research (pp. 229-325). Hillsdale, NJ: Erlbaum.

Kintsch, W., Kozminsky, E., Streby, W. J., McKoon, G., \& KeENAN, J. M. (1975). Comprehension and recall of text as a function of content variables. Journal of Verbal Learning \& Verbal Behavior, 14, 196-214

Kliegl, R., Olson, R. K., \& Davidson, B. J. (1982). Regression analysis as a tool for studying reading processes: Comments on Just and Carpenter's eye fixation theory. Memory \& Cognition, 10, 287-296.

Kučera, H., \& Francis, W. N. (1967). Computational analysis of present-day American English. Providence: Brown University Press.

MARCUS, M. P. (1980). A theory of syntactic recognition for natural language. Cambridge, MA: MTT Press.

Miller, J. R., \& KINTSCH, W. (1980). Readability and recall of short prose passages: A theoretical analysis. Journal of Experimental Psychology: Human Learning \& Memory, 6, 335-354.

Mitchell, D. C., \& GREen, D. W. (1978). The effects of context and content on immediate processing in reading. Journal of Experimental Psychology, 30, 609-636.

Olson, G. M., Mack, R. L., \& DufFy, S. A. (1981). Cognitive aspects of genre. Poetics, 10, 283-315.

Pedhazur, E. J. (1977). Coding subjects in repeated measures designs. Psychological Bulletin, 84, 298-305.

Perfetti, C. A. (1985). Reading ability. In R. J. Sternberg (Ed.), Human abilities: An information-processing approach (pp. 59-81). New York: Freeman. 
Perfetti, C. A., \& Goldman, S. R. (1976). Discourse memory and reading comprehension skill. Journal of Verbal Learning \& Verbal Behavior, 15, 33-42.

Rothkopf, E. Z., \& BilLington, M. J. (1983). Passage length and recall with test size held constant: Effects of modality, pacing, and learning set. Journal of Verbal Learning \& Verbal Behavior, 22, 667-681.

Sharkey, N. E., \&harkey, A. J. C. (1987). What is the point of integration? The loci of knowledge-based facilitation in sentence processing. Journal of Verbal Learning \& Verbal Behavior, 26, 255-276.

van Dijk, T. A., \& Kintsch, W. (1983). Strategies of discourse comprehension. New York: Academic Press.

\section{NOTES}

1. These factors were shown to be significant predictors of reading times in our previous research (see Graesser \& Haberlandt, 1986; Haberlandt \& Graesser, 1985; Haberlandt et al., 1986). Both clause-final words and sentence-final words were represented as binary variables. Thus, there was one variable for clause-final words; these were coded 1, while all other words were coded 0 . A similar variable was created for sentencefinal words. We examined the degree of collinearity between the 12 predictor variables in the current set of passages and found similar results as in our previous research (Haberlandt \& Graesser, 1985, Table 2). There were 66 bivariate correlation coefficients, with four exceeding $r^{2}=0.05$, the collinearity cutoff we used. The highest level of collinearity was found between word length and word log occurrence fre- quency $\left(r^{2}=0.56\right)$. However, previous research has shown that these two variables contribute independently to the reading-time variance (see Haberlandt \& Graesser, 1985; Just \& Carpenter, 1987, p. 46). The remaining cases of collinear variables involved the cumulative number of new arguments per sentence and sentence-serial position $\left(r^{2}=0.09\right)$, beginning of screen and beginning of line $\left(r^{2}=0.07\right)$, and end of screen and end of line $\left(r^{2}=0.07\right)$.

2. Beta coefficients reflect the relative contribution of a variable after all predictor variables have been equated for scale of measurement.

3. The subsidiary analysis involved a multiple-regression analysis in which lexical and other factors such as sentence-serial position and number of new arguments were partialled out. The purpose of this analysis was to detect interactions involving passage type. There were no interactions with passage type in an ANOVA on beta coefficients associated with the new-argument factor.

4. The absolute increase was shown in a regression analysis of mean reading times of words up to and including the first new-argument noun of each sentence. The same set of predictor variables as described in the section on intersentence integration at clause boundaries was used except for the two sentence-boundary variables. The regression coefficient for new-argument nouns occurring for the first time in a sentence was $22 \mathrm{msec}(p<.01)$, thus indicating that new arguments require an additional $22 \mathrm{msec}$ of processing time, even without the hypothetical buffering load.

(Manuscript received November 2, 1987; revision accepted for publication July 6,1988 .) 